\title{
Diffeomorphisms and approximate invariants on fuzzy sphere
}

\author{
Goro Ishiki \\ Tomonaga Center for the History of the Universe, University of Tsukuba, and \\ Graduate School of Pure and Applied Sciences, University of Tsukuba, \\ Tsukuba, Ibaraki 305-8571, Japan \\ E-mail: ishiki@het.ph.tsukuba.ac.jp

\section{Takaki Matsumoto*} \\ School of Theoretical Physics, Dublin Institute for Advanced Studies, \\ 10 Burlington Road, Dublin 4, Ireland \\ E-mail: takakiestp.dias.ie
}

\begin{abstract}
We consider how diffeomorphisms act on the configuration space of matrices in the matrix regularization. We construct the matrix regularization in terms of the Berezin-Toeplitz quantization and define diffeomorphisms on the space of matrices by using this quantization map. For the case of the fuzzy $S^{2}$, we explicitly construct the matrix version of holomorphic diffeomorphisms on $S^{2}$. We also propose three methods of constructing approximate invariants on the fuzzy $S^{2}$. These are exactly invariant under unitary similarity transformations and only approximately invariant under the general diffeomorphisms.
\end{abstract}

Corfu Summer Institute 2019 "School and Workshops on Elementary Particle Physics and Gravity" (CORFU2019)

31 August - 25 September 2019

Corfú, Greece

${ }^{*}$ Speaker. 


\section{Introduction}

The matrix regularization [ $[\mathbf{W}$, ] gives a regularization of the world volume theory of membranes. Let $\Sigma$ be a closed Riemann surface, which represents a membrane. In the light-cone gauge, the Hamiltonian of the world volume theory is written in terms of the Poisson algebra of functions on $\Sigma$ with a Poisson bracket. The matrix regularization is an operation of replacing the Poisson algebra by the Lie algebra of $N \times N$ matrices. After this replacement, the world volume theory becomes a quantum mechanical system with matrix variables and coincides with the BFSS matrix model [3] which is conjectured to give a complete formulation of M-theory in the infinite momentum frame. This coincidence suggests that the matrix regularization is not just a regularization of the world volume theory but a fundamental formulation of M-theory ${ }^{1}$.

Although the original world volume theory has the world volume diffeomorphism symmetry, it is restricted to area-preserving diffeomorphisms on $\Sigma$ in the light-cone gauge. By the matrix regularization, this residual gauge symmetry is replaced by the $U(N)$ gauge symmetry which acts on the matrix variables as unitary similarity transformations. However, we have not completely understood how general diffeomorphisms on $\Sigma$ act on the matrix variables ${ }^{2}$. Since diffeomorphisms are essential in constructing a covariant formulation of M-theory, it is important to clarify the full diffeomorphisms in the matrix model. The description of general diffeomorphisms in terms of matrices may also enable us to formulate theories of gravity on noncommutative spaces $[\mathbb{G}, \mathbb{Q}, \mathbb{Q}, \mathbb{Q}, \mathbb{Q}]$ using the matrix regularization.

In this paper, we focus on automorphisms of $C^{\infty}(\Sigma)$ induced by diffeomorphisms on $\Sigma$ rather than diffeomorphisms themselves. This is reasonable since the group of diffeomorphisms on $\Sigma$ is isomorphic to that of automorphisms of $C^{\infty}(\Sigma)$. In the matrix regularization, automorphisms of $C^{\infty}(\Sigma)$ are mapped to transformations between matrices. From this correspondence, we study how diffeomorphisms act on the space of the matrices.

For this purpose, we need to fix the scheme of the matrix regularization. A systematic scheme is given by the Berezin-Toeplitz quantization [10, ㄸ], [2] $]^{3}$, which is developed in the context of the geometric and the deformation quantizations. In this paper, we construct the matrix regularization of $S^{2}$ in terms of the Berezin-Toeplitz quantization and investigate how diffeomorphisms on $S^{2}$, which are not necessarily are-preserving, act on the space of the matrices. In particular, for holomorphic diffeomorphisms on $S^{2}$, we explicitly construct one-parameter deformations of the standard fuzzy $S^{2}$. We also propose three kinds of approximate diffeomorphism invariants on the fuzzy $S^{2}$. These are exactly invariant under the unitary similarity transformations and also invariant under general diffeomorphisms in the large- $N$ limit. These results were firstly presented in [एव]].

The organization of this paper is as follows. In section 2, we review the Berezin-Toeplitz quantization. In section 3, we define the action of diffeomorphisms on the space of matrices. In section 4, we construct the matrix regularization of $S^{2}$ based on the Berezin-Toeplitz quantization

\footnotetext{
${ }^{1}$ The matrix regularization is also applied to type IIB string theory and provides a matrix model for a nonperturbative formulation of the string theory [䧃]

${ }^{2}$ In [可], it is shown that diffeomorphisms can be embedded into the unitary transformations, if one considers the matrices as covariant derivative acting on an infinite dimensional Hilbert space. This formulation is different from the matrix regularization, which we discuss in this paper.

${ }^{3}$ The same construction was also considered in the context of the tachyon condensation on D-branes [[13], [4], [45]]

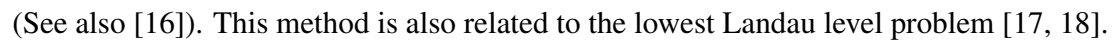


and then investigate the matrix version of holomorphic diffeomorphisms on $S^{2}$. In section 5, we propose the approximate invariants. In section 6 , we summarize our results.

\section{Berezin-Toeplitz quantization}

In this section, we briefly review the Berezin-Toeplitz quantization. In the following, we assume $\Sigma$ to be a closed Riemann surface with a symplectic form $\omega$ and denote by $\{\cdot, \cdot\}$ the poisson bracket induced by $\omega$.

The matrix regularization is formally defined by a family of linear maps from functions on $\Sigma$ to $N \times N$ matrices, $\left\{T_{N}: C^{\infty}(\Sigma) \rightarrow M_{N}(\mathbf{C})\right\}_{N \in \mathbf{N}}$, which satisfy

$$
\begin{aligned}
& \lim _{N \rightarrow \infty}\left\|T_{N}(f) T_{N}(g)-T_{N}(f g)\right\|=0, \\
& \lim _{N \rightarrow \infty}\left\|N\left[T_{N}(f), T_{N}(g)\right]-\mathrm{i} T_{N}(\{f, g\})\right\|=0,
\end{aligned}
$$

for any $f, g \in C^{\infty}(\Sigma)[20]$. Here, $\|\cdot\|$ denotes the operator norm. These conditions and the linearity of $T_{N}$ means that $T_{N}$ is approximately an algebra homomorphism and the accuracy of the approximation improves as the matrix size tends to infinity.

The Berezin-Toeplitz quantization gives a systematic way of generating the linear maps satisfying ([.]). The basic data required for the Berezin-Toeplitz quantization are zero modes of a certain Dirac operator. Our setup is as follows. We choose a metric $g$ which is compatible with $\omega$ and the standard complex structure on $\Sigma$. We denote by $e_{a}(a=1,2)$ an orthonormal frame with respect to $g$ and by $\theta^{a}$ the dual basis of $e_{a}$. We also define a spin connection $\Omega_{a b}$ by

$$
\begin{aligned}
& \Omega_{a b}+\Omega_{b a}=0, \\
& \Omega^{a}{ }_{b} \wedge \theta^{b}+d \theta^{a}=0 .
\end{aligned}
$$

Let $A$ be a $U(1)$ gauge connection such that it satisfies $d A=2 \pi \omega V^{-1}$ where $V=\int_{\Sigma} \omega$ is the symplectic volume. In this set up, we consider spinor fields with two components and define a Dirac operator acting on them by

$$
D=\mathrm{i} \sigma^{a} e_{a}^{\mu}\left(\partial_{\mu}+\frac{1}{4} \Omega_{\mu}^{a b} \sigma_{a} \sigma_{b}-\mathrm{i} N A_{\mu}\right),
$$

where $\sigma_{a}$ are the Pauli matrices and $N$ is a positive integer corresponding to the charge of the spinor fields. Then, it follows that $\operatorname{dim} \operatorname{Ker} D=N$ from the Atiyah-Singer index theorem and the vanishing theorem [2]].

Let $\psi_{i}(i=1,2, \ldots, N)$ be the orthonormal basis of $\operatorname{Ker} D$ with respect to the inner product defined by

$$
(\psi, \phi) \equiv \int_{\Sigma} \omega \psi^{\dagger} \cdot \phi
$$

where - denotes the contraction of the spinor indices. Using these zero modes, we define the socalled Toeplitz operator for $f \in C^{\infty}(\Sigma)$ by

$$
\left\langle i\left|T_{N}(f)\right| j\right\rangle=\left(\psi_{j}, f \psi_{i}\right)
$$


where $\{|i\rangle \mid i=1,2, \cdots, N\}$ is an orthonormal basis of $\mathbf{C}^{N}$ corresponding to $\psi_{i}$. In this construction,

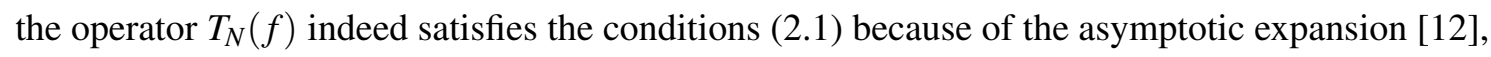

$$
T_{N}(f) T_{N}(g)=T_{N}\left(C_{0}(f, g)\right)+\frac{1}{N} T_{N}\left(C_{1}(f, g)\right)+O\left(N^{-2}\right)
$$

for any $f, g \in C^{\infty}(\Sigma)$, where $C_{0}(f, g)=f g$ and $C_{1}(f, g)-C_{1}(g, f)=\mathrm{i}\{f, g\}$.

\section{Matrix diffeomorphisms}

In this section, we define the action of diffeomorphisms in the configuration space of matrices following [ए]].

Let $\Sigma$ be a closed Riemann surface. We denote by $\operatorname{Diff}(\Sigma)$ the group of diffeomorphisms from $\Sigma$ to itself. Let $\varphi \in \operatorname{Diff}(\Sigma)$. For $f \in C^{\infty}(\Sigma), \varphi$ induces a new function on $\Sigma$ defined by

$$
\varphi^{*} f=f \circ \varphi
$$

where $\varphi^{*}$ is the pullback by $\varphi$. Then, the map $f \mapsto \varphi^{*} f$ defines an automorphism of $C^{\infty}(\Sigma)$. Inversely, an arbitrary automorphism of $C^{\infty}(\Sigma)$ is expressed in the form (B. This means that $\operatorname{Diff}(\Sigma)$ is isomorphic to the group of automorphisms of $C^{\infty}(\Sigma)^{4}$.

For the automorphism $f \mapsto \varphi^{*} f$, we define a transformation of $N \times N$ matrices by

$$
T_{N}(f) \mapsto T_{N}\left(\varphi^{*} f\right) .
$$

We call this transformation a matrix diffeomorphism corresponding to $\varphi$.

It is well-known that area-preserving diffeomorphisms are realized as unitary similarity transformations in the matrix regularization. This can also be seen by comparing the symmetries of the light-cone membrane and the matrix model. The definition (3.2) also realizes this correspondence. The infinitesimal transformation of $f$ induced by an are-preserving diffeomorphism can be written as $\delta f=\{f, \alpha\}$ with a function $\alpha$ on $\Sigma$. From ([2.6), one can see that this transformation is mapped to the infinitesimal matrix diffeomorphism,

$$
\delta T_{N}(f)=T_{N}(\delta f)=-\mathrm{i} N\left[T_{N}(f), T_{N}(\alpha)\right]+O\left(N^{-1}\right) .
$$

This is nothing but the infinitesimal form of a unitary similarity transformation.

Conversely, if (3.3]) holds, then $\delta f$ is an area-preserving diffeomorphism. This is shown as follows. Suppose that (B.3) holds for a certain $\alpha \in C^{\infty}(\Sigma)$. Then, because of (‥ 1 ), we have $T_{N}(\delta f-\{\alpha, f\})=O\left(N^{-1}\right)$. This is satisfied if and only if $\delta f-\{\alpha, f\}=0$ [ㅁ]]. Hence, $\delta f$ is area-preserving. These arguments show that for non-area-preserving diffeomorphisms, the corresponding matrix diffeomorphisms cannot be written in the form of (B.3]).

Although diffeomorphisms can be regarded as automorphisms on the space of functions, matrix diffeomorphisms are not necessarily an automorphism of $M_{N}(\mathbf{C})$, which can always be written as a similarity transformation. This is because the Toeplitz operator is not an isomorphism from $C^{\infty}(\Sigma)$ to $M_{N}(\mathbf{C})$. In fact, the definition (B.2) contains a much broader class of transformations than the similarity transformations. In the next section, we will explicitly construct some of those transformations for fuzzy $S^{2}$.

\footnotetext{
${ }^{4}$ See e.g. Section 1.3 in [ㄹ] for a precise proof.
} 


\section{Matrix diffeomorphisms on fuzzy sphere}

In this section, we consider the Berezin-Toeplitz quantization and matrix diffeomorphisms on the fuzzy $S^{2}$ [[2]]. We will explicitly construct matrix diffeomorphisms on the fuzzy $S^{2}$ corresponding to holomorphic diffeomorphisms and see that most of them can not be written as a similarity transformation.

\subsection{Berezin-Toeplitz quantization on $S^{2}$}

First, we construct the Berezin-Toeplitz quantization map for $S^{2}$. We identify $S^{2}$ with the Riemann sphere $\hat{\mathbf{C}}=\mathbf{C} \cup\{\infty\}$ and cover it by two open subsets $U_{z} \equiv \hat{\mathbf{C}}-\{\infty\}$ and $U_{w} \equiv \hat{\mathbf{C}}-\{0\}$. As a local coordinate on $U_{z}$, we choose the stereographic coordinate,

$$
z \equiv \frac{x^{1}+\mathrm{i} x^{2}}{1+x^{3}}
$$

where $x^{A}(A=1,2,3)$ are the embedding coordinates from $S^{2}$ to $\mathbf{R}^{3}$ which satisfy the equation $\sum_{A=1}^{3} x^{A} x^{A}=1$. We also define a local coordinate on $U_{w}$ by $w \equiv 1 / z$. Then the coordinate transformation is given by a holomorphic map $z \mapsto 1 / z$.

We define a symplectic form $\omega$ on $S^{2}$ by

$$
\omega=\mathrm{i} \frac{d z \wedge d \bar{z}}{\left(1+|z|^{2}\right)^{2}}
$$

such that $V=\int_{S^{2}} \omega=2 \pi$. We also define the standard complex structure $J$ by $J\left(\partial_{z}\right)=\mathrm{i} \partial_{z}$ and $J\left(\partial_{\bar{z}}\right)=-\mathrm{i} \partial_{\bar{z}}$ and define a metric $g$ by the compatible condition $g(\cdot, \cdot)=\omega(\cdot, J \cdot)$ as

$$
g=2 \frac{d z d \bar{z}}{\left(1+|z|^{2}\right)^{2}}
$$

We also need a topologically nontrivial configuration of the $U(1)$ gauge connection on $S^{2}$ to construct Toeplitz operators. We use the Wu-Yang monopole configuration,

$$
A^{(z)}=-\frac{\mathrm{i}}{2} \frac{\bar{z} d z-z d \bar{z}}{1+|z|^{2}}
$$

for $U_{z}$. On the overlap region $U_{z} \cap U_{w}$, the gauge connection $A^{(w)}$ on $U_{w}$ is related to (4.4) by a $U(1)$ gauge transformation, $A^{(w)}=A^{(z)}-d \arg (z)$. This gauge connection satisfies $d A^{(z)}=2 \pi \omega V^{-1}$.

In this set up, the Dirac operator ([2.3) for $S^{2}$ is given by

$$
D=\left(\begin{array}{cc}
0 & D^{-} \\
D^{+} & 0
\end{array}\right)
$$

where the local form of $D^{+}$and $D^{-}$on $U_{z}$ are

$$
\begin{aligned}
& D^{+}=\sqrt{2} \mathrm{i}\left\{\left(1+|z|^{2}\right) \partial_{\bar{z}}+\frac{N-1}{2} z\right\}, \\
& D^{-}=\sqrt{2} \mathrm{i}\left\{\left(1+|z|^{2}\right) \partial_{z}-\frac{N+1}{2} \bar{z}\right\},
\end{aligned}
$$


respectively.

In order to construct Toeplitz operators, we need the zero modes of $D^{ \pm}$. We can easily solve the eigenvalue equations $D^{ \pm} \psi^{ \pm}=0$ and obtain $\psi^{ \pm}=\left(1+|z|^{2}\right)^{\mp(N \mp 1) / 2} h^{ \pm}$, where $h^{+}$and $h^{-}$are arbitrary holomorphic and anti-holomorphic functions on $U_{z}$, respectively. Note that the integral,

$$
\int_{S^{2}} \omega\left|\psi^{-}\right|^{2}=\mathrm{i} \int_{S^{2}} d z d \bar{z}\left(1+|z|^{2}\right)^{(N-1)}\left|h^{-}\right|^{2},
$$

does not converge for $N \geq 1$, unless $h^{-}=0$. Thus, we find that $\operatorname{Ker} D^{-}=\{0\}$ for $N \geq 1$. The similar integral for $\psi^{+}$converges when the degree of $h^{+}$is smaller than $N$. Such $h^{+}$is a holomorphic polynomial of degree $N-1$, which can be expanded in terms of the basis $1, z, z^{2}, \ldots, z^{N-1}$. Therefore, we find that ${ }^{5} \operatorname{dimKer} D^{+}=N$. The Dirac zero modes can be written as

$$
\psi_{i}(z, \bar{z})=\sqrt{\frac{N}{2 \pi}}\left(\begin{array}{c}
\langle i \mid z\rangle \\
0
\end{array}\right),
$$

where $\{|i\rangle \mid i=1,2, \cdots, N\}$ is an arbitrary orthonormal basis of $\mathbf{C}^{N}$, and $|z\rangle$ is the Bloch coherent state with $J=(N-1) / 2$ defined by

$$
|z\rangle=\frac{1}{\left(1+|z|^{2}\right)^{J}} \sum_{r=-J}^{J} z^{J-r}\left(\begin{array}{c}
2 J \\
J+r
\end{array}\right)^{1 / 2}|J r\rangle .
$$

Here, $\{|J r\rangle \mid r=-J,-J+1, \ldots, J\}$ is the standard basis of the $(2 J+1)$-dimensional irreducible representation space of $S U(2)$. By using the resolution of identity, $N \int_{S^{2}} \omega|z\rangle\langle z| / 2 \pi=\mathbf{1}_{N}$, one can check that $\left\{\psi_{i} \mid i=1,2, \cdots, N\right\}$ is an orthonormal basis of $\operatorname{Ker} D$.

In the above setup, the Toeplitz operators (2.5T) are written as

$$
\left\langle i\left|T_{N}(f)\right| j\right\rangle=\frac{N}{2 \pi} \int_{S^{2}} \omega\langle i \mid z\rangle f(z, \bar{z})\langle z \mid i\rangle .
$$

Let us focus on the embedding coordinates $x^{A}$ from $S^{2}$ to $\mathbf{R}^{3}$, which are smooth real valued functions on $S^{2}$. From (لـ), we have

$$
\begin{aligned}
& x^{1}=\frac{z+\bar{z}}{1+|z|^{2}}, \\
& x^{2}=\frac{i(\bar{z}-z)}{1+|z|^{2}}, \\
& x^{3}=\frac{1-|z|^{2}}{1+|z|^{2}} .
\end{aligned}
$$

It is easy to find that the Toeplitz operators of $x^{A}$ are given by

$$
T_{N}\left(x^{A}\right)=\frac{L^{A}}{J+1},
$$

where $L^{A}$ are the $N$-dimensional irreducible representation of the generators of $S U(2)$. This is the well-known configuration of the fuzzy $S^{2}$.

\footnotetext{
${ }^{5}$ Note that these results are consistent with the vanishing theorem and the index theorem, $\operatorname{Ind} D=N$.
} 


\subsection{Holomorphic matrix diffeomorphisms}

We consider the matrix diffeomorphisms (3.2) for $X^{A} \equiv T_{N}\left(x^{A}\right)$. Since there are infinitely many diffeomorphisms even for the simple manifold $S^{2}$, we restrict ourselves to the holomorphic diffeomorphisms in the following.

Any holomorphic diffeomorphism on $S^{2}$ is expressed as a Möbius transformation, which is defined by

$$
\varphi(z)=\frac{a z+b}{c z+d}
$$

where $a, b, c, d$ are complex numbers such that $a d-b c \neq 0^{6}$. We define $\varphi(\infty)=\infty$ for $c=0$ and $\varphi(\infty)=a / c$ for $c \neq 0$. Since multiplying $a, b, c, d$ by a common number does not change the value of (4.[3]), we can fix $a d-b c=1$. We focus on the four special transformations,

$$
\begin{aligned}
R_{\theta}(z) & =e^{\mathrm{i} \theta} z, \\
D_{\lambda}(z) & =e^{\lambda} z, \\
T_{\eta}(z) & =z+\eta, \\
S_{\zeta}(z) & =\frac{z}{\zeta z+1},
\end{aligned}
$$

where $\theta, \lambda \in \mathbf{R}$ and $\eta, \zeta \in \mathbf{C}$. These are a rotation, dilatation, translation and special conformal transformation, respectively. Note that any Möbius transformation can be constructed as the composition of these special transformations ${ }^{7}$. Note also that $R_{\theta}$ preserves the symplectic form (4.2) and the compatible metric (4.3), while the other three transformations are neither isometries nor area-preserving diffeomorphisms. We consider one-parameter groups, $\left\{R_{t \theta}\right\}_{t \in \mathbf{R}},\left\{D_{t \lambda}\right\}_{t \in \mathbf{R}}$, $\left\{T_{t \eta}\right\}_{t \in \mathbf{R}}$ and $\left\{S_{t \zeta}\right\}_{t \in \mathbf{R}}$, which generate the vector fields

$$
\begin{aligned}
& u_{R}=\mathrm{i} \theta\left(z \partial_{z}-\bar{z} \partial_{\bar{z}}\right), \\
& u_{D}=\lambda\left(z \partial_{z}+\bar{z} \partial_{\bar{z}}\right), \\
& u_{T}=\eta \partial_{z}+\bar{\eta} \partial_{\bar{z}}, \\
& u_{S}=-\zeta z^{2} \partial_{z}-\bar{\zeta} \bar{z}^{2} \partial_{\bar{z}},
\end{aligned}
$$

respectively.

For a diffeomorphism generated by a vector field $u$, the infinitesimal variation of the embedding function $x^{A}$ is given by the Lie derivative $L_{u} x^{A}$. Correspondingly, the variation of the matrices are given by $\delta X^{A}=T_{N}\left(L_{u} x^{A}\right)$. Let $X^{ \pm}=T_{N}\left(x^{ \pm}\right)=T_{N}\left(x^{1} \pm \mathrm{i} x^{2}\right)$. After some calculations, we easily

\footnotetext{
${ }^{6}$ The condition $a d-b c \neq 0$ ensures that $\varphi$ is not a constant function. For $a d-b c=0$, we have $\varphi(z)=b / d$.

${ }^{7}$ In fact, for $c=0$, the Möbius transformation is linear and is given by a composition of $R_{\theta}, D_{\lambda}$ and $T_{\eta}$. For $c \neq 0$, it is expressed as $\varphi(z)=\left(T_{(a-1) / c} \circ S_{c} \circ T_{(d-1) / c}\right)(z)$.
} 
find that the infinitesimal variations of $X^{A}$ for the vector fields (4. 45 ) are given by [प]]

$$
\begin{aligned}
& \delta_{R} X^{+}=i \theta X^{+} \\
& \delta_{R} X^{-}=-i \theta X^{-} \\
& \delta_{R} X^{3}=0 \\
& \delta_{D} X^{+}=\lambda X^{3} X^{+}+O\left(N^{-1}\right) \\
& \delta_{D} X^{-}=\lambda X^{3} X^{-}+O\left(N^{-1}\right) \\
& \delta_{D} X^{3}=-\lambda X^{+} X^{-}+O\left(N^{-1}\right) \\
& \delta_{T} X^{+}=\frac{1}{2} \eta\left(\mathbf{1}_{N}+X^{3}\right)^{2}-\frac{1}{2} \bar{\eta}\left(X^{+}\right)^{2}+O\left(N^{-1}\right), \\
& \delta_{T} X^{-}=\frac{1}{2} \bar{\eta}\left(\mathbf{1}_{N}+X^{3}\right)^{2}-\frac{1}{2} \eta\left(X^{-}\right)^{2}+O\left(N^{-1}\right), \\
& \delta_{T} X^{3}=-\frac{1}{2}\left(\mathbf{1}_{N}+X^{3}\right)\left(\bar{\eta} X^{+}+\eta X^{-}\right)+O\left(N^{-1}\right) \\
& \delta_{S} X^{+}=\frac{1}{2} \bar{\zeta}\left(\mathbf{1}_{N}-X^{3}\right)^{2}-\frac{1}{2} \zeta\left(X^{+}\right)^{2}+O\left(N^{-1}\right), \\
& \delta_{S} X^{-}=\frac{1}{2} \zeta\left(\mathbf{1}_{N}-X^{3}\right)^{2}-\frac{1}{2} \bar{\zeta}\left(X^{-}\right)^{2}+O\left(N^{-1}\right), \\
& \delta_{S} X^{3}=\frac{1}{2}\left(\mathbf{1}_{N}-X^{3}\right)\left(\zeta X^{+}+\bar{\zeta} X^{-}\right)+O\left(N^{-1}\right) .
\end{aligned}
$$

The rotation (4.16) can be written as $\delta_{R} X^{A}=-\mathrm{i} N\left[X^{A}, \theta X^{3} / 2\right]+O\left(N^{-1}\right)$. This is the infinitesimal transformation of a unitary similarity transformation. We also notice that the other three matrix diffeomorphisms are not unitary similarity transformations. For example, let us check the case of $\delta_{D} X^{A}$. If $\delta_{D} X^{3}$ is a similarity transformation, we have $\delta_{D} X^{3} \propto\left[U, X^{3}\right]$ with $U$ a certain matrix. Then, we will have

$$
\left\langle J r\left|\delta_{D} X^{3}\right| J r\right\rangle=0
$$

for all $r$. However, $\left\langle J r\left|\delta_{D} X^{3}\right| J r\right\rangle=-\lambda(J+r)(J-r+1) /(J+1)^{2}$ is not zero for $r \neq-J$. Thus, the matrix diffeomorphism corresponding to $D_{t \lambda}$ is not a similarity transformation.

Our definition of the matrix diffeomorphisms also works for finite transformations. As an example, let us consider the dilatation. The finite diffeomorphism transforms of (4.]) are given by

$$
\begin{aligned}
& D_{t \lambda}^{*} x^{1}=\frac{e^{t \lambda}(z+\bar{z})}{1+e^{2 t \lambda}|z|^{2}}, \\
& D_{t \lambda}^{*} x^{2}=\frac{i e^{t \lambda}(\bar{z}-z)}{1+e^{2 t \lambda}|z|^{2}}, \\
& D_{t \lambda}^{*} x^{3}=\frac{1-e^{2 t \lambda}|z|^{2}}{1+e^{2 t \lambda}|z|^{2}} .
\end{aligned}
$$

In the following, we set $\lambda=1$ and $t \geq 0$ for simplicity. For example, the matrix elements of $T_{N}\left(D_{t}^{*} x^{3}\right)$ with respect to the basis $|J r\rangle$ reduces to the following integral,

$$
I \equiv \int_{S^{2}} \omega \frac{z^{J-r} \bar{z}^{J-r^{\prime}}}{\left(1+|z|^{2}\right)^{2 J}} \frac{1-e^{2 t}|z|^{2}}{1+e^{2 t}|z|^{2}} .
$$


After integrating out the argument of $z$ and exchanging the integral variable from $|z|^{2}$ to $y=1 /(1+$ $|z|^{2}$ ), we obtain

$$
\begin{aligned}
I=2 \pi & \delta_{r r^{\prime}}\left(1+e^{-2 t}\right) \int_{0}^{1} d y y^{J+r+1}(1-y)^{J-r}\left\{1-\left(1-e^{-2 t}\right) y\right\}^{-1} \\
& -2 \pi \delta_{r r^{\prime}} \int_{0}^{1} d y y^{J+r}(1-y)^{J-r}\left\{1-\left(1-e^{-2 t}\right) y\right\}^{-1}
\end{aligned}
$$

For a while, we suppose that $t \neq 0$. For $t>0$, we have $\left|1-e^{-2 t}\right|<1$. Using the integral representation of Gauss's hyper geometric function $F(\alpha, \beta, \gamma ; s)$ for $|s|<1$ and $0<\alpha<\gamma$,

$$
F(\alpha, \beta, \gamma ; s)=\frac{\Gamma(\gamma)}{\Gamma(\alpha) \Gamma(\gamma-\alpha)} \int_{0}^{1} d y y^{\alpha-1}(1-y)^{\gamma-\alpha-1}(1-s y)^{-\beta}
$$

we can rewrite (4.23) as

$$
\begin{gathered}
I=2 \pi \delta_{r r^{\prime}}\left(1+e^{-2 t}\right) \frac{\Gamma(J+r+2) \Gamma(J-r+1)}{\Gamma(2 J+3)} F\left(J+r+2,1,2 J+3 ; 1-e^{-2 t}\right) \\
-2 \pi \delta_{r r^{\prime}} \frac{\Gamma(J+r+1) \Gamma(J-r+1)}{\Gamma(2 J+2)} F\left(J+r+1,1,2 J+2 ; 1-e^{-2 t}\right) .
\end{gathered}
$$

The calculations of the Toeplitz operators for $D_{t}^{*} x^{+}$and $D_{t}^{*} x^{-}$also reduce to similar integrals. After

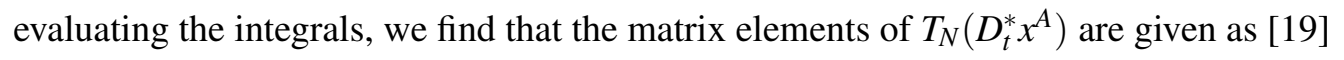

$$
\begin{aligned}
&\left\langle J r\left|T_{N}\left(D_{t}^{*} x^{+}\right)\right| J r^{\prime}\right\rangle=\delta_{r-1 r^{\prime}} \frac{e^{-t}}{J+1} \sqrt{(J-r+1)(J+r)} F\left(J+r+1,1,2 J+3 ; 1-e^{-2 t}\right), \\
&\left\langle J r\left|T_{N}\left(D_{t}^{*} x^{-}\right)\right| J r^{\prime}\right\rangle=\delta_{r+1 r^{\prime}} \frac{e^{-t}}{J+1} \sqrt{(J+r+1)(J-r)} F\left(J+r+2,1,2 J+3 ; 1-e^{-2 t}\right), \\
&\left\langle J r\left|T_{N}\left(D_{t}^{*} x^{3}\right)\right| J r^{\prime}\right\rangle= \delta_{r r^{\prime}} \frac{1}{2(J+1)}\left\{\left(1+e^{-2 t}\right)(J+r+1) F\left(J+r+2,1,2 J+3 ; 1-e^{-2 t}\right)\right. \\
&\left.-2(J+1) F\left(J+r+1,1,2 J+2 ; 1-e^{-2 t}\right)\right\} .
\end{aligned}
$$

Since $F(\alpha, \beta, \gamma: 0)=1$, we have $T_{N}\left(D_{0}^{*} x^{A}\right)=X^{A}$. Thus, the supposition of $t \neq 0$ can be removed.

Again, we can check that the map $X^{A} \mapsto T_{N}\left(D_{t}^{*} x^{A}\right)$ is not a unitary similarity transformation by comparing the eigenvalue set of $T_{N}\left(D_{t}^{*} x^{3}\right)$ and that of $X^{3}$ numerically [प्प].

\section{Approximate diffeomorphism invariants}

In this section, we construct three kinds of approximate invariants for the matrix diffeomorphisms on the fuzzy $S^{2}$ proposed in [ㅍ] . These are functions $I(X)$ of the Toeplitz operators $X^{A}=T_{N}\left(x^{A}\right)$ which satisfy

$$
I(X+\delta X)=I(X)+O\left(N^{-1}\right),
$$

for any infinitesimal matrix diffeomorphism $\delta X$ on the fuzzy $S^{2}$. In particular, if $\delta X$ is an infinitesimal unitary transformation, then they satisfy $I(X+\delta X)=I(X)$. 


\subsection{Invariants from matrix Dirac operator}

For $N \times N$ matrices $X^{A}(A=1,2,3)$ and the embedding function $x^{A}$ defined in (4.T), we define a Dirac type operator,

$$
\hat{D}=\sum_{A=1}^{3} \sigma^{A} \otimes\left(X^{A}-\hat{x}^{A}\right) .
$$

Here, we put a hat on $x^{A}$ to emphasize that $\hat{x}^{A}$ are kept fixed when we discuss the variation of approximate invariants, (5.]) $\left(\hat{x}^{A}\right.$ are equal to $x^{A}$ as functions, $\left.\hat{x}^{A}=x^{A}\right)$. We also introduce the eigenstates of $\hat{D}$ as

$$
\hat{D}|n\rangle=E_{n}|n\rangle,
$$

where the eigenvalues shall be labeled such that $\left|E_{0}\right| \leq\left|E_{1}\right| \leq\left|E_{2}\right| \leq \cdots$. Note that $\hat{D},|n\rangle$ and $E_{n}$ depend on local coordinates on $S^{2}$ through $\hat{x}^{A}$, although the dependences are not written explicitly. Apart from the fixed embedding function, the operator (5.2) depends only on the matrices $X^{A}$. In this sense, $E_{n}$ and $|n\rangle$ are functions of $X_{A}$. The eigenvalues $E_{n}$ are not invariant for general transformations of matrices $X^{A} \mapsto X^{\prime} A$, but are exactly invariant under the unitary similarity transformations.

In the following, we consider the case in which $X^{A}$ are given by the Toeplitz operators of the embedding function (4.1). By solving the eigenvalue problem for this case [24, 255, [26], one can find that $E_{0}$ and $|0\rangle$ are given by

$$
\begin{aligned}
& E_{0}=\frac{J}{J+1}-1=O\left(N^{-1}\right), \\
& |0\rangle=U_{2}\left(\begin{array}{l}
1 \\
0
\end{array}\right) \otimes|z\rangle .
\end{aligned}
$$

Here, $U_{2}=e^{z \sigma^{-}} e^{-\sigma^{3} \log \left(1+|z|^{2}\right)} e^{-\bar{z} \sigma^{+}}$is a local rotation matrix and $|z\rangle$ is the Bloch coherent state (4.9).

The eigenvalue $E_{0}$, which has the smallest absolute value, gives our first example of the approximate invariants. Under an infinitesimal variation $X^{A} \mapsto X^{A}+\delta X^{A}, E_{0}$ transforms as ${ }^{8}$

$$
\delta E_{0}=\sum_{A=1}^{3}\left\langle 0\left|\sigma^{A} \otimes \delta X^{A}\right| 0\right\rangle .
$$

We again emphasize that here $\hat{x}^{A}$ are kept fixed and we consider only the variation of the matrices. Now, suppose that $\delta X^{A}$ is given by a matrix diffeomorphism, which can be written as

$$
\delta X^{A}=\frac{N}{2 \pi} \int_{S^{2}} \omega|w\rangle \delta x^{A}(w)\langle w|,
$$

where $\delta x^{A}$ is the variation of $x^{A}$ under a diffeomorphism. Then, (5.5) is evaluated as

$$
\delta E_{0}=\sum_{A=1}^{3} x^{A} \delta x^{A}+O\left(N^{-1}\right)
$$

\footnotetext{
${ }^{8}$ This is just the first order formula of the perturbation theory in quantum mechanics.
} 
In deriving (5.7), the following property of the Bloch coherent state is useful:

$$
\begin{aligned}
|\langle z \mid w\rangle|^{2} & =\frac{|1+w \bar{z}|^{4 J}}{\left(1+|z|^{2}\right)^{2 J}\left(1+|w|^{2}\right)^{2 J}} \\
& =\exp \left[2 J \log \left\{1-\frac{|z-w|^{2}}{\left(1+|z|^{2}\right)\left(1+|w|^{2}\right)}\right\}\right], \\
& =\frac{\pi}{2 J}\left(1+|z|^{2}\right)^{2} \delta^{(2)}(z-w)+O\left(N^{-2}\right) .
\end{aligned}
$$

Since $\sum_{A} x^{A} x^{A}=1$, the first term of (5.]) is vanishing. Thus, $E_{0}$ is indeed invariant under the matrix diffeomorphism up to the $1 / N$ corrections.

In [27], it was proposed that the matrix Dirac operator can be used to find effective shapes of fuzzy branes. Here, the loci of the zero eigenvalue of the matrix Dirac operator are identified with the effective shape embedded in the flat target space. See also [24, 28, 20]. The same method was also independently proposed in the context of the tachyon condensation in string theory [144, [15], 25]].

These invariants have the information of the induced metric for the embedding $\hat{x}^{A}$. As shown in [BO]], by considering variations of $\hat{x}^{A}$, we can construct from $E_{0}$ the Levi-Civita connection and the Riemann curvature tensor for the induced metric.

\subsection{Invariants of information metric}

By using the eigenstate $|0\rangle$ defined in the previous subsection, we introduce a density matrix,

$$
\rho=|0\rangle\langle 0|
$$

This gives an embedding of $S^{2}$ into the space of density matrices [26]. Then, the pullback $h$ of the so-called information metric in the space of density matrices,

$$
h_{\mu v} d \sigma^{\mu} d \sigma^{v}=\operatorname{Tr} d \rho d \rho
$$

gives a metric structure on $S^{2}$, where $\sigma^{\mu}$ are the local coordinates on $S^{2}$.

In our setup, the definition of $h$ depends on the choice of $X^{A}$ and $\hat{x}^{A}$. However, in the setup of [D]], $\hat{x}^{A}$ are just thought of as three real parameters and the structure of embedding appears after solving the eigenvalue problem. The underlying space can be defined as the loci of zeros of the matrix Dirac operator. In this sense, the definition of $h$ depends only on the matrices $X^{A}$ and it gives a good geometric object defined in terms of the matrix variables.

Note that $h$ is exactly invariant under unitary similarity transformations $X^{A} \mapsto U^{\dagger} X^{A} U$. Below, we show that the information metric is also approximately covariant under general matrix diffeomorphisms. First, because $E_{0} \rightarrow 0(N \rightarrow \infty)$, we have $\left\langle 0\left|\hat{D}^{2}\right| 0\right\rangle \rightarrow 0$. This implies that $\left(X^{A}-\hat{x}^{A}\right)|0\rangle \rightarrow 0$ for $A=1,2,3$. Let $\delta x^{A}$ be a polynomial of $x^{A}$ with the degree much less than $N$. Then, we also have

$$
\left(\delta X^{A}-\delta x^{A}\right)|0\rangle \rightarrow 0
$$

as $N \rightarrow \infty$, where $\delta X^{A}$ is the Toeplitz operator of $\delta x^{A}$. Let $\delta x^{A}$ be a Lie derivative of $x^{A}$ and $\delta X^{A}$ the corresponding matrix diffeomorphism. Under the matrix diffeomorphism $X^{A} \mapsto X^{A}+\delta X^{A}$, the 
state $|0\rangle$ transforms as

$$
\begin{aligned}
\delta|0\rangle & =\sum_{n \neq 0} \sum_{A=1}^{3} \frac{|n\rangle\left\langle n\left|\sigma^{A} \otimes \delta X^{A}\right| 0\right\rangle}{E_{0}-E_{n}}+\mathrm{i} \delta \lambda|0\rangle, \\
& =\sum_{n \neq 0} \sum_{A=1}^{3} \frac{|n\rangle\left\langle n\left|\sigma^{A}\right| 0\right\rangle \delta x^{A}}{E_{0}-E_{n}}+\mathrm{i} \delta \lambda|0\rangle+O\left(N^{-1}\right),
\end{aligned}
$$

where $\delta \lambda$ is a real number and we used (D. I) to obtain the last expression. We again emphasize that we fix $\hat{x}^{A}$ and consider only the variation of $X^{A}$. On the other hand, from the infinitesimal variation of the local coordinates, we obtain

$$
\partial_{\mu}|0\rangle=-\sum_{n \neq 0} \sum_{A=1}^{3} \frac{|n\rangle\left\langle n\left|\sigma^{A}\right| 0\right\rangle \partial_{\mu} x^{A}}{E_{0}-E_{n}}+\mathrm{i} A_{\mu}|0\rangle,
$$

where $A=-\mathrm{i}\langle 0|d| 0\rangle$ is the Berry connection. For a diffeomorphism $\delta x^{A}=u^{\mu} \partial_{\mu} x^{A}$, from (5.12) and $(5 \cdot 3)$, we find

$$
\delta \rho=-u^{\mu} \partial_{\mu} \rho+O\left(N^{-1}\right) .
$$

This means that the embedding function $\rho$ transforms as a scalar field under matrix diffeomorphisms. Thus, the induced metric $h$ is also covariant:

$$
\delta h_{\mu v}=-\nabla_{\mu} u_{v}-\nabla_{v} u_{\mu}+O\left(N^{-1}\right) .
$$

Diffeomorphism invariants (in the usual sense) defined in terms of $h$ are also approximately invariant under matrix diffeomorphisms. For example, the volume integral $\int_{S^{2}} \sqrt{h}$ or the EinsteinHilbert action $\int_{S^{2}} \sqrt{h} R$ gives an approximate invariant.

In general, the information metric is different from the induced metric discussed in the previous subsection. For Kähler manifolds, the information metric gives a Kähler metric compatible with the field strength of the Berry connection [B] ] and hence has intrinsic information on the manifold, which does not depend on the embedding.

\subsection{Heat kernel on fuzzy sphere}

For a $2 n$-dimensional closed Riemannian manifold $(M, g)$, the heat kernel,

$$
K(t)=\operatorname{Tr} e^{-t \Delta}
$$

for the Laplacian, $\Delta=-(1 / \sqrt{g}) \partial_{\mu}\left(\sqrt{g} g^{\mu v} \partial_{v}\right)$, generates diffeomorphism invariants on $M$ as coefficients of the asymptotic expansion in $t \rightarrow+0$ :

$$
K(t)=\frac{1}{(4 \pi t)^{n}} \int_{M} \sqrt{g}+\frac{1}{(4 \pi)^{n} t^{n-1}} \int_{M} \sqrt{g} \frac{R}{6}+\cdots .
$$

Similarly, we define the heat kernel on the fuzzy $S^{2}$ as

$$
\hat{K}\left(t_{N}, p\right)=\operatorname{Tr} e^{-t_{N} \hat{\Delta}} .
$$


Here, $\hat{\Delta}$ is the matrix version of the Laplacian defined by

$$
\hat{\Delta}=(J+1)^{2} \sum_{A=1}^{3}\left[X^{A},\left[X^{A}, \cdot\right]\right]=\sum_{A=1}^{3}\left[L^{A},\left[L^{A}, \cdot\right]\right],
$$

where $X^{A}=T_{N}\left(x^{A}\right)$ is given in (A.D2). See [B2, [3] ] for the properties of $\hat{K}$ for finite size matrices.

It is well-known that the spectrum of $\hat{\Delta}$ coincides with that of the standard Laplacian on $S^{2}$ up to a UV cutoff given by the matrix size. The eigenstates of $\hat{\Delta}$ are given by the fuzzy spherical

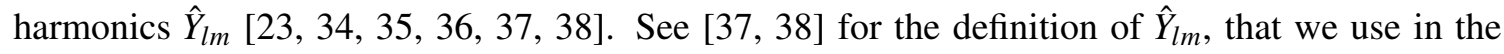
following. For $\hat{Y}_{l m}, l$ runs from 0 to $N-1$ and $m$ runs from $-l$ to $l$. The eigenvalue of $\hat{\Delta}$ is $l(l+1)$ for $\hat{Y}_{l m}$, which coincides with the spectrum of the spherical harmonics on $S^{2}$, except that the angular momentum $l$ has a cutoff $N-1$ for the fuzzy spherical harmonics.

For finite $N$, the spectrum of $\hat{\Delta}$ is finite. Thus, the matrix heat kernel (5.]l) has only a regular expansion in $t_{N} \rightarrow+0$ as $\hat{K}=\operatorname{Tr} \mathbf{1}_{N^{2}}+O\left(t_{N}\right)$, which looks trivial and seems not to have any interesting information of the geometry. However, it is obvious that if we first take the large- $N$ limit and then take $t_{N} \rightarrow+0, \hat{K}$ should behave similarly to $K$ having a singular expansion. In other words, by putting $t_{N}=N^{-\alpha}$, where $\alpha$ is a small positive number, the heat kernel should have the expansion,

$$
\hat{K}\left(t_{N}=N^{-\alpha}, N\right)=\frac{1}{t_{N}} c_{0}+c_{1}+O\left(t_{N}\right)
$$

in the large- $N$ limit. It follows from the Euler-Maclaurin formula that the coefficients are given by $c_{0}=1$ and $c_{1}=1 / 3$ for the Laplacian ([.]) . The values of $c_{0}$ and $c_{1}$ just coincide with the coefficients of the heat kernel expansion (5.J]) for $S^{2}$. Thus, in the double scaling limit, the matrix heat kernel possesses geometric information of $S^{2}$.

Now, we show that the matrix heat kernel $(5.18)$ is approximately invariant under matrix diffeomorphisms. Let us consider a perturbation $X^{A} \mapsto X^{A}+\delta X^{A}$. Let $\delta X^{A}$ be a general infinitesimal matrix for the moment. (In the end of the calculation, we will restrict $\delta X^{A}$ to be a matrix diffeomorphism.) The eigenvalues of $\hat{\Delta}$ are perturbed by $\delta X^{A}$. Let $\delta_{l m}$ be the deviation of the eigenvalue for the mode $\hat{Y}_{l m}$. From the first order formula of the perturbation theory, one obtains that

$$
\delta_{l m}=\frac{(J+1)}{N} \operatorname{Tr} \sum_{A=1}^{3}\left(\hat{Y}_{l m}^{\dagger}\left[\delta X^{A},\left[L^{A}, \hat{Y}_{l m}\right]\right]+\hat{Y}_{l m}^{\dagger}\left[L^{A},\left[\delta X^{A}, \hat{Y}_{l m}\right]\right]\right) .
$$

The heat kernel (5.18) changes by

$$
\delta \hat{K}=-t_{N} \sum_{l=0}^{N-1} \sum_{m=-l}^{l} e^{-t_{N} l(l+1)} \delta_{l m} .
$$

The matrix $\delta X^{A}$ can be expanded in terms of the vector fuzzy spherical harmonics as

$$
\delta X^{A}=\sum_{l=0}^{N-1} \sum_{m=-l}^{l} \sum_{\rho=-1}^{1} \delta X_{l m \rho} \hat{Y}_{l m \rho}^{A} .
$$

Again, see [37, [38] for the definition of $\hat{Y}_{l m \rho}^{A}$. After an easy calculation, we find that (5.22) is given as

$$
\delta \hat{K}=2 \mathrm{i} t_{N} \delta X_{00-1} \sqrt{\frac{J+1}{J}} \sum_{l=0}^{N-1} e^{-t_{N} l(l+1)} l(l+1)(2 l+1)
$$


The important point is that the $\delta \hat{K}$ depends only on $\delta X_{00-1}$. This is exactly the mode proportional to $L^{A 9}$. This mode changes the radius of $S^{2}$ in the target space, and $\sum_{A}\left(X^{A}+\delta X^{A}\right)^{2}$ will deviate from the identity matrix even in the large- $N$ limit. Here, any matrix diffeomorphism should keep the relation

$$
\sum_{A=1}^{3} X^{A} X^{A}=\mathbf{1}_{N}+O\left(N^{-1}\right)
$$

corresponding to the constraint $\sum_{A} x^{A} x^{A}=1$ since any diffeomorphism on $S^{2}$ does not break this constraint. The fluctuation of $\delta X_{00-1}$ violates this constraint, so it is not a matrix diffeomorphism. Therefore, for matrix diffeomorphisms, the matrix heat kernel is invariant. The coefficients in the expansion (5.20) give approximate invariants on fuzzy $S^{2}$.

The matrix Laplacian corresponds to the operator $-\sum_{A}\left\{x^{A},\left\{x^{A}, \cdot\right\}\right\}$, because of (2. operator can be written as $-g^{v \sigma} \partial_{v} \partial_{\sigma}+\cdots$, where $g^{v \sigma}=W^{\mu v} W^{\rho \sigma} \sum_{A}\left(\partial_{\mu} x^{A} \partial_{\rho} x^{A}\right)$ and $W^{\mu v}$ is the Poisson tensor. The (inverse) metric $g^{v \sigma}$ is the open string metric [B]] in the strong magnetic flux. Thus, the invariants from the heat kernel are associated with the open string metric.

\section{Summary}

In this paper, we defined the action of diffeomorphisms on the space of matrices through the matrix regularization following [ㅁ] . We first constructed the matrix regularization based on the Berezin-Toeplitz quantization and then defined the matrix diffeomorphisms as the matrix regularization of automorphisms of functions induced by diffeomorphisms. We also studies holomorphic matrix diffeomorphisms on the fuzzy $S^{2}$ and constructed three kinds of approximate invariants of the matrix diffeomorphisms on it. They are associated with three different kinds of metrics, the induced metric, Kähler metric and open string metric. Although, they are equivalent up to an overall factor in the case of $S^{2}$, this is not the case for general spaces as shown in [B], 26]].

\section{Acknowledgments}

We thank N. Ishibashi and P. V. Nair for valuable discussions and encouraging comments. The work of G. I. was supported, in part, by Program to Disseminate Tenure Tracking System, MEXT, Japan and by KAKENHI (16K17679).

\section{References}

[1] J. Hoppe, "Quantum theory of a relativistic surface and a two-dimensional bound state ploblem," Ph. D. thesis. MIT (1982)

[2] B. de Wit, J. Hoppe and H. Nicolai, Nucl. Phys. B 305, 545 (1988).

[3] T. Banks, W. Fischler, S. H. Shenker and L. Susskind, Phys. Rev. D 55, 5112 (1997).

[4] N. Ishibashi, H. Kawai, Y. Kitazawa and A. Tsuchiya, Nucl. Phys. B 498, 467 (1997).

[5] M. Hanada, H. Kawai and Y. Kimura, Prog. Theor. Phys. 114, 1295 (2006).

\footnotetext{
${ }^{9}$ Namely, if we consider a perturbation such that $\delta X_{l m \rho} \propto \delta_{l 0} \delta_{m 0} \delta_{\rho-1}$, such $\delta X^{A}$ is proportional to $L^{A}$.
} 
[6] A. H. Chamseddine and A. Connes, Commun. Math. Phys. 186, 731 (1997).

[7] P. Aschieri, M. Dimitrijevic, F. Meyer and J. Wess, Class. Quant. Grav. 23, 1883 (2006).

[8] H. Steinacker, Class. Quant. Grav. 27, 133001 (2010).

[9] V. P. Nair, Phys. Rev. D 92, no. 10, 104009 (2015).

[10] S. Klimek and A. Lesniewski, Comm. Math. Phys. 146, no. 1, 103-122 (1992).

[11] M. Bordemann, E. Meinrenken and M. Schlichenmaier, Commun. Math. Phys. 165, 281 (1994).

[12] X. Ma and G. Marinescu, J. Geom. Anal. 18, 565-611 (2008).

[13] T. Asakawa, S. Sugimoto and S. Terashima, JHEP 0203, 034 (2002).

[14] S. Terashima, JHEP 0510, 043 (2005).

[15] S. Terashima, JHEP 1807, 008 (2018).

[16] I. Ellwood, JHEP 0508, 078 (2005).

[17] K. Hasebe, Int. J. Mod. Phys. A 31, no. 20n21, 1650117 (2016).

[18] K. Hasebe, Nucl. Phys. B 934, 149 (2018).

[19] G. Ishiki and T. Matsumoto, PTEP 2020, no. 1, 013 B04 (2020).

[20] J. Arnlind, J. Hoppe and G. Huisken, J. Diff. Geom. 91, no. 1, 1 (2012).

[21] X. Ma and G. Marinescu, Math. Z. 240, no. 3, 651-664 (2002).

[22] J. M. Gracia-Bondia, J. C. Várilly and H. Figueroa, "Elements of Noncommutative Geometry," Birkhäuser, Boston (2001).

[23] J. Madore, Class. Quant. Grav. 9, 69 (1992).

[24] M. H. de Badyn, J. L. Karczmarek, P. Sabella-Garnier and K. H. C. Yeh, JHEP 1511, 089 (2015).

[25] T. Asakawa, G. Ishiki, T. Matsumoto, S. Matsuura and H. Muraki, PTEP 2018, no. 6, 063 B04 (2018).

[26] G. Ishiki, T. Matsumoto and H. Muraki, Phys. Rev. D 98, no. 2, 026002 (2018).

[27] D. Berenstein and E. Dzienkowski, Phys. Rev. D 86, 086001 (2012).

[28] J. L. Karczmarek and K. H. C. Yeh, JHEP 1511, 146 (2015).

[29] L. Schneiderbauer and H. C. Steinacker, J. Phys. A 49, no. 28, 285301 (2016).

[30] G. Ishiki, Phys. Rev. D 92, no. 4, 046009 (2015).

[31] G. Ishiki, T. Matsumoto and H. Muraki, JHEP 1608, 042 (2016).

[32] N. Sasakura, JHEP 0412, 009 (2004).

[33] N. Sasakura, JHEP 0503, 015 (2005).

[34] H. Grosse, C. Klimcik and P. Presnajder, Commun. Math. Phys. 178, 507 (1996).

[35] S. Baez, A. P. Balachandran, B. Ydri and S. Vaidya, Commun. Math. Phys. 208, 787 (2000).

[36] K. Dasgupta, M. M. Sheikh-Jabbari and M. Van Raamsdonk, JHEP 0205, 056 (2002).

[37] G. Ishiki, S. Shimasaki, Y. Takayama and A. Tsuchiya, JHEP 0611, 089 (2006).

[38] T. Ishii, G. Ishiki, S. Shimasaki and A. Tsuchiya, Phys. Rev. D 78, 106001 (2008).

[39] N. Seiberg and E. Witten, JHEP 9909, 032 (1999). 